\title{
Relativistic surfatron process for Landau resonant electrons in radiation belts
}

\author{
A. Osmane ${ }^{1,2}$ and A. M. Hamza ${ }^{1}$ \\ ${ }^{1}$ University of New Brunswick, Physics Department, Fredericton, New Brunswick, Canada \\ ${ }^{2}$ Aalto University, Radio Science and Engineering, Espoo, Finland
}

Correspondence to: A. Osmane (a.osmane@unb.ca)

Received: 22 July 2013 - Revised: 9 October 2013 - Accepted: 12 December 2013 - Published: 16 January 2014

\begin{abstract}
Recent theoretical studies of the nonlinear waveparticle interactions for relativistic particles have shown that Landau resonant orbits could be efficiently accelerated along the mean background magnetic field for propagation angles $\theta$ in close proximity to a critical propagation $\theta_{\mathrm{c}}$ associated with a Hopf-Hopf bifurcation condition. In this report, we extend previous studies to reach greater modeling capacities for the study of electrons in radiation belts by including longitudinal wave effects and inhomogeneous magnetic fields. We find that even though both effects can limit the surfatron acceleration of electrons in radiation belts, gains in energy of the order of $100 \mathrm{keV}$, taking place on one tenth of a millisecond, are sufficiently strong for the mechanism to be relevant to radiation belt dynamics.
\end{abstract}

\section{Introduction}

The following report aims at extending a theoretical model for wave-particle coherent interaction (Osmane and Hamza, 2012a; Osmane and Hamza, 2012b) to characterize electron dynamics in the Earth's radiation belts. Electrons with energies in the $\mathrm{MeV}$ range have been frequently measured in the inner and outer component of the radiation belt. An increase in relativistic electron flux observed for short time scales (from few hours to few days) and in correlation with an increase of magnetic activity during the recovery phase of geomagnetic substorms (Friedel et al., 2002; O'brien et al., 2003). Not only relativistic electrons constitute a threat to satellites and spacecrafts in orbit, but their production has to be understood in order to account for the magnetospheric energy budget. Thus far, numerous theoretical models have been proposed. They can be grouped into two categories: mechanisms relying on radial transport alone as well as those that rely on internal mechanisms such as wave-particle interactions and recirculation models (see, e.g., Friedel et al., 2002 and reference therein, Albert, 2002; Shprits et al., 2006; Summers and Omura, 2007). Radial transport is often described as a diffusion mechanism driven by the fluctuations in the large scale magnetosphere electric and magnetic fields. As the particles spread from the outer magnetosphere to smaller equatorial radial distances, $L$, the first two adiabatic invariants are conserved while the third one is violated, resulting in an increase of energy (Kulsrud, 2005). On the other hand, local wave-particle interactions, and other local mechanisms accelerate particles already present in the inner magnetosphere. Even though it is accepted that radial diffusion is an important transport mechanism, particle energization on time scales shorter than the drift period predicted by radial diffusion has lead to hold wave-particle interaction responsible for a number of observables. Events occurring on time scale of days are believed to originate from electromagnetic cyclotron waves and whistler waves through pitch angle scattering, whereas the more intense and monochromatic chorus waves are believed to be the source of the strong energy increase occurring on the shortest time scales (Miyoshi et al., 2002; Thorne et al., 2005; Albert, 2002; Summers and Omura, 2007). More recently, observations of peaks of phase space density, in contradiction with inward radial diffusion, have shown that wave-particle interactions are dominant mechanisms (Green and Kivelson, 2004; Horne et al., 2005; Chen et al., 2007).

Aside from the outstanding problem of flux enhancement of relativistic electrons in the radiation belt, wave-particle interaction could also be proven to hold a decisive role in a number of other magnetospheric problems. Among them lies 
the generation of relativistic electron microbursts observed in association with VLF chorus waves (Lorentzen et al., 2001; Summers and Omura, 2007; Omura Hikishima et al., 2010) as well as precipitation rates of electrons entering the loss cone in pitch angle.

Perhaps more importantly, the most recent waveforms measured in the radiation belts have revealed additional motivating reasons to consider the wave-particle interaction as a dominating energy-momentum exchange mechanism in radiation belt problems. Large-amplitude, monochromatic, obliquely propagating, and bursty waveforms were not only repeatedly measured in the radiation belt (Catell et al., 2008; Kellog et al., 2010; Kersten et al., 2011; Wilson III et al., 2011), but appeared correlated with electron energization (Wilson III et al., 2011) as well as relativistic microbursts events (Kersten et al., 2011). The correlation between chorus waves and electron energization in the radiation belts is not recent, but it is suspected that if such waveforms were more commonly present in the radiation belts they could be the dominant trigger responsible for the energization of electrons on short timescales. A study by Yoon (2011) has shown that if one solves the plasma equations self-consistently, such waveforms were indeed capable of accelerating electrons on kinetic time scales consistent with the observations. Even though our study lacks the levels of self-consistency provided by the numerical method developed by Yoon (2011), we will show hereafter that we arrive at similar conclusions if we choose parameters consistent with the radiation beltmeasured waveforms.

The large-amplitude wave forms are observed with a longitudinal component and the analysis above needs to be conducted with the addition of this compressive electric component. Whereas the addition of the electrostatic field with the same phase as the electromagnetic components of the fields would result in the same condition for the surfatron process, a difference in phase would shift the Hopf bifurcation and have non-trivial effects that need to be scrutinized. Moreover, radiation belt electrons are confined in the magnetic field of the Earth, and one must take into account the effect of field inhomogeneities. We therefore proceed in this in this report by modifying a previously derived dynamical system (Osmane and Hamza, 2012a) to allow a study of relativistic electrons in radiation belts.

\section{Longitudinal effects}

We follow the procedure described in Osmane and Hamza (2012a); Osmane and Hamza (2012b) for the derivation of a dynamical system to study the interaction of an ion with an obliquely propagating wave composed of a transverse and longitudinal component. As shown in Fig. 1, the electromagnetic wave is composed of a transverse component along the $(\hat{x}, \hat{y})$ plane and a longitudinal component along the $\hat{z}$

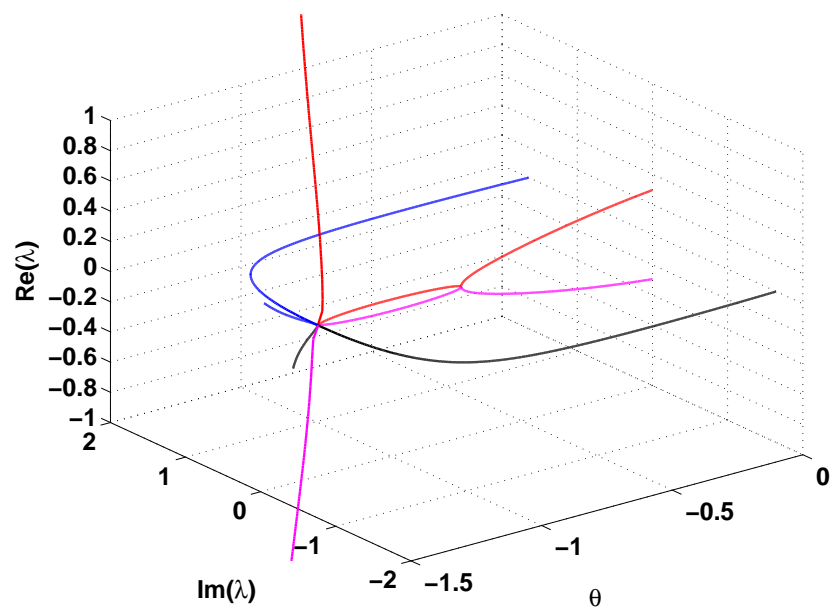

Fig. 1. Electromagnetic field configuration. A circularly polarized wave propagating obliquely to a background magnetic field $\mathbf{B}_{0}$ with a longitudinal component.

direction superposed onto a background magnetic field $B_{0}$ in the $(\hat{y}, \hat{z})$ plane:

$\boldsymbol{E}(\boldsymbol{x}, t)=\boldsymbol{\delta} \boldsymbol{E}_{\mathrm{em}}(\boldsymbol{x}, t)+\boldsymbol{\delta} \boldsymbol{E}_{k}(\boldsymbol{x}, t)$

$\boldsymbol{B}(\boldsymbol{x}, t)=\boldsymbol{B}_{0}+\delta \mathbf{B}(\boldsymbol{x}, t)$.

We write the longitudinal component as a function of parameters $\eta$ and $\Psi$ as follows:

$\boldsymbol{\delta} \boldsymbol{E}_{k}(\boldsymbol{x}, t)=\eta \delta E \sin (k \boldsymbol{z}-\omega t+\Psi) \hat{z}$.

The parameter $\eta$ quantify the amplitude of the longitudinal component of the wave with respect to the electromagnetic component. Setting $\eta=1$ would therefore result in having equal electric field amplitudes parallel and perpendicular to the wave vector $\boldsymbol{k}$. Setting $\eta=0$ recovers the case treated in Osmane and Hamza (2012b). The parameter $\Psi$ is a phase difference between the longitudinal component and the transverse component. It is added for the sake of completeness. The dynamical system equation is therefore not fundamentally modified. The difference resides in the addition of an electric field component along the $z$ component of the Lorentz force. It is easy to show that the dynamical system takes the following form (Osmane and Hamza, 2012b) (see Appendix for detailed derivation of the purely transverse case):

$\left\{\begin{aligned} \dot{p}_{x}^{\prime}= & \Omega_{0} p_{y}^{\prime} \cos (\theta)-\Omega_{1} p_{z}^{\prime} \cos \left(k z^{\prime}\right)+\Omega_{0}\left(p_{z}^{\prime}+p_{\phi}\right) \sin (\theta) \\ \dot{p}_{y}^{\prime}= & -\Omega_{0} p_{x}^{\prime} \cos (\theta)+\Omega_{1} p_{z}^{\prime} \sin \left(k z^{\prime}\right) \\ \dot{p}_{z}^{\prime}= & -p_{x}^{\prime} \Omega_{0} \sin (\theta)+\Omega_{1} \frac{n^{2}-1}{n^{2}}\left(p_{x} \cos \left(k z^{\prime}\right)-p_{y}^{\prime} \sin \left(k z^{\prime}\right)\right) \\ & -\frac{1}{n^{2}} \Omega_{1} \eta p_{z}^{\prime} \sin \left(k z^{\prime}+\Psi\right)+\Omega_{1} \frac{n^{2}-1}{n^{2}} \eta p_{\phi} \sin \left(k^{\prime}+\Psi\right) \\ \dot{z}^{\prime}= & p_{z}^{\prime} v_{\Phi} / p_{\Phi}\end{aligned}\right.$

for the dynamical gyrofrequencies $\Omega_{0}=\frac{e B_{0}}{m \gamma c}$ and $\Omega_{1}=\frac{e \delta B}{m \gamma c}$, relativistic momentum $p_{i}=m \gamma v_{i}^{\prime}$, refractive index $n=\frac{c}{v_{\phi}}$, phase speed $v_{\Phi}=\omega / k$ and dots indicating time derivatives. 
The time evolution of the dynamical gyrofrequency can then be written as:

$$
\begin{aligned}
\frac{d \Omega_{0}}{\mathrm{~d} t} & =\frac{-\Omega_{0} \Omega_{1} p_{\Phi}}{m^{2} \gamma^{2} c^{2}}\left(p_{x}^{\prime} \cos \left(k z^{\prime}\right)-p_{y}^{\prime} \sin \left(k z^{\prime}\right)\right. \\
& \left.+\eta\left(p_{\phi}+p_{z}^{\prime}\right) \sin \left(k z^{\prime}+\Psi\right)\right) .
\end{aligned}
$$

We can now proceed by studying the dynamical system properties in terms of the fixed points and their stability as well as the dependence on wave parameters such as wave obliquity $\theta$ and normalized wave amplitude $\delta_{1}=\delta B / B_{0}$. More importantly, we would like to know whether the surfatron acceleration mechanism, the processes by which a particle is trapped along the wave vector and accelerated uniformly along the background magnetic field by the parallel electric field (Osmane and Hamza, 2012b), is also available when a longitudinal component is added. The surfatron is a trapping effect, and is therefore only achievable if a parallel electric field of sufficiently large amplitude is present. If the propagation of the wave is parallel, the parallel electric field is zero, and neither trapping along the wave vector nor acceleration along the background magnetic field is possible. We therefore assume that the longitudinal component can for various parameters either enhance or destroy the surfatron.

A quick look at the dynamical system (Eq. 3) shows that the fixed point for the electromagnetic case $\left(p_{x}^{\prime}=p_{z}^{\prime}=0\right.$, $\left.p_{y}^{\prime}=-p_{\phi} \tan (\theta), k z^{\prime}=0\right)$ exists for $\Psi=0$. For $\Psi \neq 0$ no fixed points exist. Hence, one would expect the acceleration mechanisms associated with Hopf-Hopf bifurcation to be available for sufficiently large wave amplitude capable of trapping when $\Psi=0$. Whenever $\Psi \neq 0$, one can think of the longitudinal component as a perturbation to uniformly accelerated particles (orbits). Hence, particles (orbits) could still be energized, but the longitudinal component could break the locking eventually. Additionally, if the longitudinal component of the wave, for given parameters $\eta$ and $\Psi$, cancels the parallel component of the electric field with respect to the background magnetic field due to the electromagnetic component, no uniform acceleration should take place. This condition can be written as follows:

$\delta \mathbf{E} \cdot \mathbf{b}_{0}=-\delta E_{y} \sin (\theta)+\delta E_{k} \cos (\theta)=0$.

Setting $\Psi=0$, one finds that this condition translates to $\eta=-\tan (\theta)$, for which neither trapping nor uniform acceleration should be possible. We therefore expect the lack of a parallel electric field component to translate into unstable orbits for linear perturbation around the fixed points. In the next section we apply the stability analysis to the dynamical system (Eq. 3) for the fixed point $\left(p_{x}^{\prime}=p_{z}^{\prime}=0, p_{y}^{\prime}=-p_{\phi} \tan (\theta)\right.$, $\left.k z^{\prime}=0\right)$.

\subsection{Stability analysis}

We now proceed similarly as for the electromagnetic case (Osmane and Hamza, 2012b) to quantify the linear stability of the fixed point ${ }^{1}$. In order to do so we linearize the dynamical system and assume a perturbation of the form $\sum_{i=1}^{4} \xi_{i} e^{\lambda_{i} t}$, for which an imaginary eigenvalue translates into marginally stable orbits, negative eigenvalues into linearly stable orbits, and positive eigenvalues linearly into unstable orbits. Computing the resulting Jacobian evaluated at the fixed point $\mathbf{J}=\left.\frac{\partial F}{\partial x_{i}}\right|_{\mathbf{x}_{0}}$ for the longitudinal case, we find the following matrix:

$\mathbf{J}=\left(\begin{array}{llll}0 & a & b & 0 \\ c & 0 & 0 & 0 \\ d & 0 & 0 & e \\ 0 & 0 & f & 0\end{array}\right)$

for the parameters $\delta_{1}, \delta_{2}=\frac{m \omega c}{e B_{0}}, \theta$ and $\eta$ :

$$
\begin{aligned}
& a=\frac{\cos (\theta)}{\delta_{2} \gamma_{0}}\left(1-\frac{\tan (\theta)^{2}}{n^{2}-1}\right) \\
& b=\frac{\mp \delta_{1}+\sin (\theta) \frac{n^{2}}{n^{2}-1}}{\delta_{2} \gamma_{0}} \\
& c=-\frac{\cos (\theta)}{\delta_{2} \gamma_{0}} \\
& d=\left(-\sin (\theta) \pm \delta \frac{n^{2}-1}{n^{2}}\right) \frac{1}{\delta_{2} \gamma_{0}} \\
& e=\mp \frac{\delta_{1}}{\delta_{2}} \frac{n^{2}-1}{n^{2}}(\tan (\theta)+\eta)-\lambda \\
& f=\frac{1}{\gamma_{0}}=\sqrt{1-\frac{v_{\Phi}^{2}}{c^{2}}\left(1+\tan ^{2}(\theta)\right) .}
\end{aligned}
$$

Once again the dark \pm and $\mp$ correspond to the fixed points components for $Z=(0, \pi)$, that is the upper sign for $Z=0$ and the lower one for $Z=\pi$. Hence, all four fixed points are represented in this Jacobian matrix and their stability can be analyzed by choosing the right \pm symbols. In order to find the eigenvalues, we need to solve the characteristic polynomial given by the following expression:

$$
\chi(\lambda)=\left|\begin{array}{llll}
-\lambda & \frac{\cos (\theta)}{\delta_{2} \gamma_{0}}\left(1-\frac{\tan (\theta)^{2}}{n^{2}-1}\right) \frac{\mp \delta_{1}+\sin (\theta) \frac{n^{2}}{\delta_{2}-1}}{\delta_{2} \gamma_{0}} & 0 \\
-\frac{\cos (\theta)}{\delta_{20} \gamma_{0}} & -\lambda & 0 & 0 \\
\left(-\sin (\theta) \pm \delta_{1} \frac{n^{2}-1}{n^{2}}\right) \frac{1}{\delta_{2} \gamma_{0}} & 0 & \mp & \mp \frac{\delta_{1}}{\delta_{2}} \frac{n^{2}-1}{n^{2}}(\tan (\theta)+\eta) \\
0 & 0 & \frac{1}{\gamma_{0}} & -\lambda
\end{array}\right|
$$

A little algebra results in the following bi-quadratic expression:

$\chi(\lambda)=\lambda^{4}+\zeta_{1} \lambda^{2}+\zeta_{2}=0$

with the values $\zeta_{1}$ and $\zeta_{2}$ given by the following expressions:

\footnotetext{
${ }^{1}$ The fixed points are located by setting the four equations of motions as zero and resolving the remaining algebraic equations.
} 


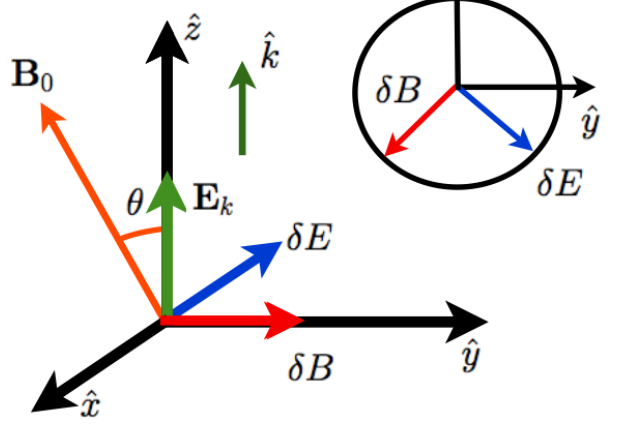

Fig. 2. Eigenvalues' dependence on the propagation angle $\theta$ for fixed parameters $\delta_{1}=0.07, \delta_{2}=0.21, n^{2}=2, \eta=0.9$ and the fixed point of component $Z_{0}=0$. A Hopf-Hopf bifurcation takes place for $\tan \left(\theta_{\mathrm{c}}\right)^{2}=n^{2}-1$ and a second bifurcation takes place for $\eta=-\tan (\theta)$, corresponding to a null parallel electric field. The fixed point is stable for $\theta<\theta_{\mathrm{c}}$ and $\eta>-\tan (\theta)$ and unstable for $\theta>\theta_{\mathrm{c}}$ and $\eta>-\tan (\theta)$.

$$
\begin{aligned}
\zeta_{1} & =\frac{\delta_{1}}{\delta_{2} \gamma_{0}} \frac{n^{2}-1}{n^{2}}(\tan (\theta)+\eta) \\
& +\frac{1}{\delta_{2}^{2} \gamma_{0}^{2}}\left(\delta_{1} \frac{n^{2}-1}{n^{2}}+1 \mp 2 \delta_{1} \sin (\theta)\right) \\
\zeta_{2} & =\frac{\delta_{1}}{\delta_{2}^{3} \gamma_{0}^{5}} \frac{n^{2}-1}{n^{2}}\left(\sin (\theta) \cos (\theta)+\eta \cos (\theta)^{2}\right) .
\end{aligned}
$$

One can compare the Jacobian matrix as well as the characteristic equation for the purely electromagnetic case with the expressions above for an additional longitudinal component with $\Psi=0$. It is clear that minimal differences arise, as denoted in the appearance of a factor of $\eta$ in the Jacobian and the characteristic equation. The characteristic equation once again has four roots given by the following equation:

$\lambda_{1,2,3,4}= \pm \sqrt{\frac{-\zeta_{1} \pm \sqrt{\zeta_{1}^{2}-4 \zeta_{2}}}{2}}$.

Figure 2 shows the dependence of all four eigenvalue solutions for typical parameters relevant to space plasmas on the propagation angle. It is clear that the Hopf-Hopf bifurcation takes place once again for parameters resulting in $\gamma_{0}=0$. That is, whenever parameters are such that $n^{2}-1=\tan ^{2}(\theta)$, the fixed point evolves from marginally stable to linearly unstable when we add a longitudinal component with $\psi=0$. Additionally, a second bifurcation takes place when $\eta=-\tan (\theta)$. Setting $\eta=-\tan (\theta)$ in the coefficients $\zeta_{1}$ and $\zeta_{2}$ results in the following characteristic equation: $\lambda^{4}+\zeta_{1} \lambda^{2}=0$. With $\zeta_{1}>0$ for $\delta_{1} \sim O(1)$, it is clear that two eigenvalues are null and two eigenvalues are imaginary. As noted in the previous section, this expression denotes a null parallel electric field resulting in the destructive interference of the parallel longitudinal component and the parallel electromagnetic component. We
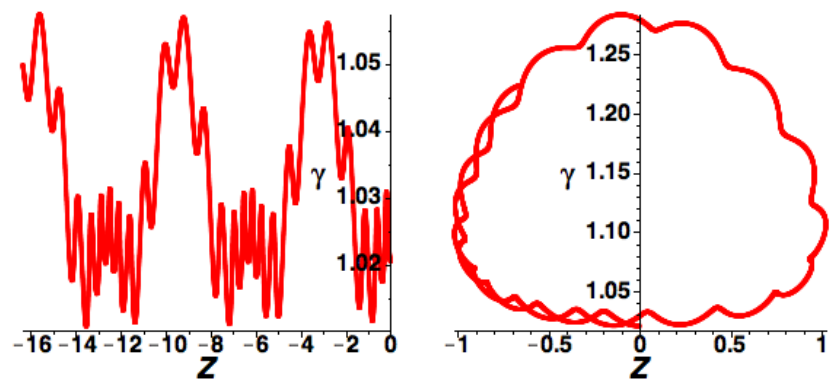

Fig. 3. Orbit for parameters $\delta_{1}=0.1, v_{\phi}=0.33 c, \theta=-31^{\circ}, \delta_{2}=0.1$ for $\eta>-\tan (\theta)$ (right panel) and $\eta<-\tan (\theta)$ (left panel). A transition from untrapped to trapped orbit is observed as we evolve the parameter $\eta$.

now investigate the nonlinear effects of the longitudinal components on the surfatron process for various parameters $\eta$ and $\psi$.

\subsection{Landau resonant orbits}

In this section we determine whether the addition of the longitudinal component enhances or prevents the uniform acceleration for orbits caught in the basin of attraction centered at Landau resonance. As noted in the stability analysis, a HopfHopf bifurcation does indeed take place when a longitudinal component is added. The main difference in the linear stability around the fixed points resides in the addition of a parallel electric field capable of canceling the electromagnetic component parallel to the background field. Hence, whenever $\eta=-\tan (\theta)$, the parallel component of the electric field is zero and the surfatron process cannot take place. Indeed, choosing the parameter $\eta$ to coincide with $\sqrt{n^{2}-1}$ results in reducing the Hopf-Hopf bifurcation to a single Hopf bifurcation (one pair of imaginary eigenvalues crossing the real plane instead of two pairs). For such a parameter the surfatron process is not expected to be applicable because a parallel electric field causing the uniform acceleration is now set to a null value.

Figure 3 shows two orbits for $\eta>-\tan (\theta)$ (in the right panel) and $\eta<-\tan (\theta)$ (in the left panel). A transition from untrapped to trapped orbit is observed as we evolve the parameter $\eta$. For $\eta=\tan (\theta)$ the particle located sufficiently close to the fixed point (Landau resonant velocity) is trapped, but small perturbation results in untrapped orbits.

In Fig. 4, the four panels represent a seeded particle with energy of the order of $100 \mathrm{keV}$ but for $\theta=40^{\circ}$ (up and left), $\theta=55^{\circ}$ (up and right), $\theta=70^{\circ}$ (down and left) and $\theta=85^{\circ}$ (down and right). The particle gains a maximum amount of energy for $\theta=70^{\circ}$, which corresponds to a propagation angle close to $\theta_{\mathrm{c}}$ and to the surfatron process. The longitudinal component for parameters $\delta_{1}=0.045, v_{\phi}=0.33 c$, $\eta=-1$, and $\delta_{2}=0.1$ enhances the parallel electric field component and results in smaller range of values in $\delta_{1}=\delta B / B_{0}$ 

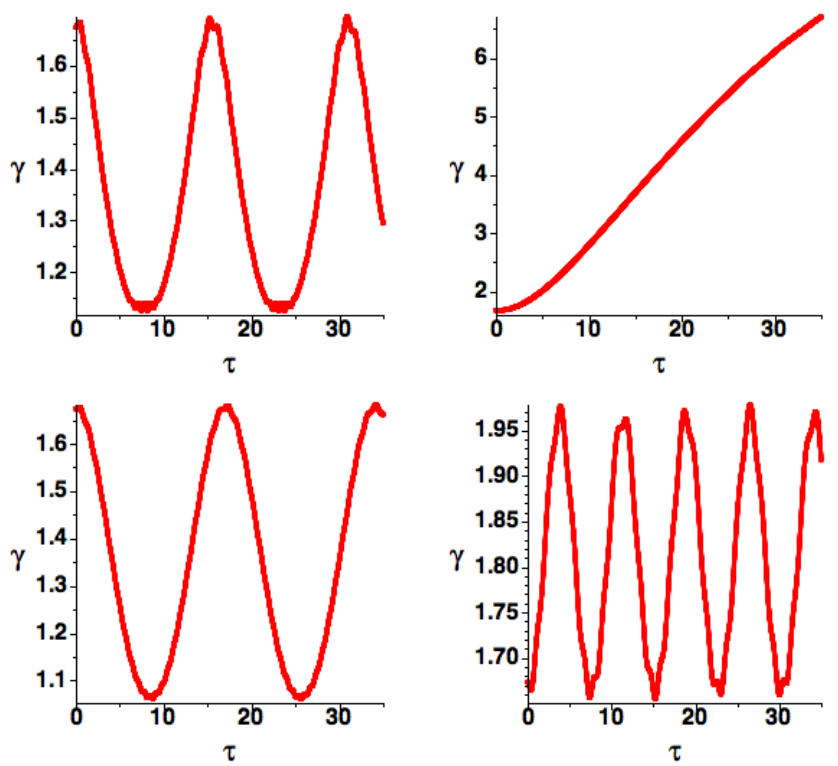

Fig. 4. Orbit for parameters $\delta_{1}=0.045, v_{\phi}=0.33 c, \eta=-1, \delta_{2}=0.1$ and $\theta=40^{\circ}$ (up and left), $\theta=55^{\circ}$ (up and right), $\theta=70^{\circ}$ (down and left) and $\theta=85^{\circ}$ (down and right). Each case is seeded with a particle of energy of the order of $100 \mathrm{keV}$. The particle gains a maximum amount of energy for $\theta=70^{\circ}$, which corresponds to a propagation angle close to $\theta_{\mathrm{c}}$. The longitudinal component can enhance the parallel electric field component and result in a smaller range of values $\delta_{1}=\delta B / B_{0}$ for which the surfatron process is accessible. In this case the ratio $\delta_{1} \sim 4 \%$.

for which the surfatron process is accessible. In this case the ratio $\delta_{1}$ is of the order of $4 \%$. This order of magnitude for the electromagnetic wave component is comparable to large-amplitude bursty waves observed in the radiation belts (Catell et al., 2008; Kellog et al., 2010; Kersten et al., 2011; Wilson III et al., 2011).

However, before concluding that the longitudinal component preserves the surfatron mechanism for $\theta=\theta_{\mathfrak{c}}$, we need to evaluate the effects of the phase difference $\Psi$. In Fig. 4 the parameter $\Psi$ has been set to zero. Yet, when $\Psi \neq 0$, the dynamical system possesses no fixed points and the acceleration observed for the surfatron process should not arise uniformly. Panels in Fig. 5 show particles for $\eta=1, \delta_{1}=0.06$, $\delta_{2}=0.1, n^{2}=9$, and $\theta=-71^{\circ}$ and three different values for $\Psi=-\pi / 4,0$ and $\pi / 4$. It is seen that depending on the phase difference, the surfatron process can still take take place for sufficiently long time to energize the particle. As for the purely electromagnetic case, a charged particle in this field would gain a significant amount of energy (from $\mathrm{keV}$ levels to $\mathrm{MeV}$ ) on small kinetic time scales $\omega t \sim 0.1 \Omega_{0} t \sim 10$. Hence a particle can be energized in such a field on time scales of the order of the $1 / 100$ of a second for a wave frequency $\omega \sim 3 \mathrm{kHz}$. Since the acceleration takes place on very small time scales, inhomogeneous effects should not prevent the mechanism entirely. We therefore conclude this section by suggesting that the large-amplitude electromagnetic waves observed in the radiation belts can energize particles efficiently on kinetic time scales for propagation angles close to the critical Hopf-Hopf bifurcation value $\theta_{\mathrm{c}}$. If the propagation angle is not sufficiently close to $\theta_{\mathrm{c}}$, then the particle will just oscillate back and forth in the potential of the electric field and no significant gain in energy should be observed. We now proceed in the next section by quantifying the inhomogeneous effects on the surfatron herein described.

\section{Inhomogeneous magnetic field effects on the surfatron}

In this section we want to include the effects of a nonhomogeneous magnetic field on the acceleration process described in the previous section. We first discuss the motion of a particle in an inhomogeneous magnetic field with no waveparticle interaction. We then provide an approximation for the time scales for which inhomogeneous effects can result in surfatron breaking and a discussion on numerical integration of a particle trajectory interacting with a large-amplitude electromagnetic wave in an inhomogeneous magnetic field.

\subsection{Particle orbits in an inhomogeneous magnetic field}

Relativistic electrons trapped in the radiation belts bounce back and forth along the (approximately) dipolar magnetic field of the Earth. Before addressing the more complicated motion of relativistic electrons bouncing back and forth in the Earth's magnetic field and at the same time interacting with an obliquely propagating wave, we would like to quantify the impact of the magnetic field inhomogeneities on the relativistic motion. Assuming that the magnetic moment

$\mu=\frac{p_{\perp}^{2}}{2 B}=\frac{m c^{2}\left(\gamma^{2}-1\right) \sin (\alpha)^{2}}{2 B}$

is an adiabatic invariant, we can derive the forces due to the magnetic field inhomogeneities as follows.

The magnetic force perpendicular to the magnetic field can be deduced from the conservation of the magnetic moment:

$\dot{p}_{\perp}=m \gamma \mu \dot{B} / p_{\perp}$.

Replacing $\mu$ in terms of $B$ and $p_{\perp}$, and $\dot{B}=v_{\|} \nabla_{\|} B$, we find the following expression for the force perpendicular to the magnetic field:

$\dot{p}_{\perp}=\frac{p_{\|} p_{\perp} \nabla_{\|} B}{2 m B \gamma}$.

Using the above equation and assuming that the energy of the particle is conserved to first order in $\mu$ for a particle moving in an inhomogeneous magnetic field, we can write:

$\gamma \dot{\gamma}=p_{\|} \dot{p}_{\|}+p_{\perp} \dot{p}_{\perp}=0$. 

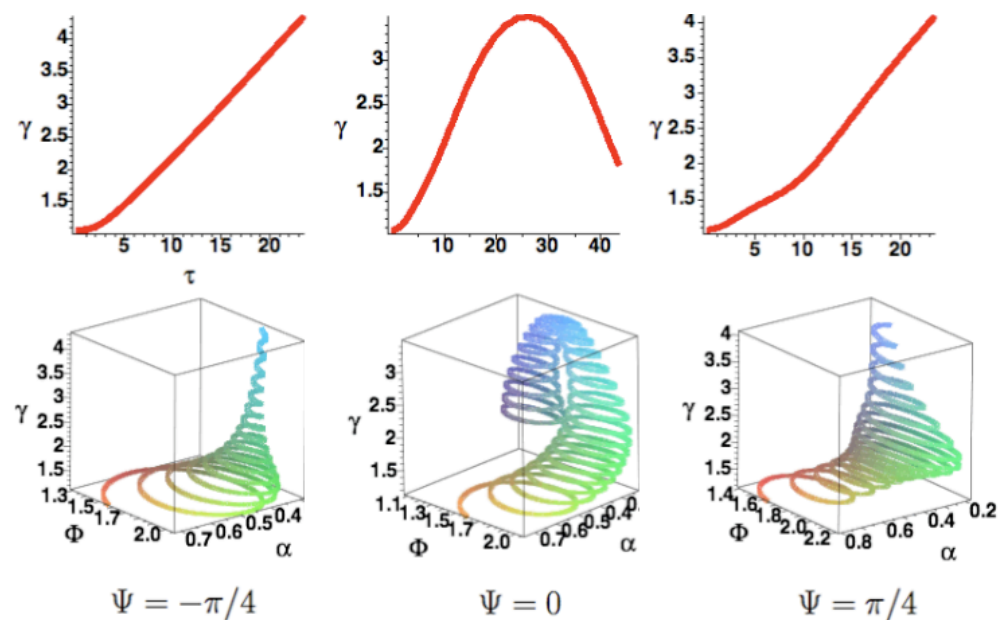

Fig. 5. Effect of the phase angle $\psi$ for the longitudinal component on the surfatron process for parameters $\left(\delta_{1}=0.06, \delta_{2}=0.1, n^{2}=9\right.$, $\left.\theta=-71^{\circ}\right)$. The phase angle for a given parameter $\eta$ determines whether the longitudinal parallel component is enabling or breaking the locking of particles into the surfatron.

Therefore, the equation of motion in the parallel direction can be written as:

$$
\begin{aligned}
\dot{p}_{\|} & =-p_{\perp} \dot{p}_{\perp} / p_{\|} \\
& =-\frac{p_{\perp}^{2} \nabla_{\|} B}{2 m B \gamma} \\
& =-\mu \nabla_{\|} B .
\end{aligned}
$$

Setting $\gamma=1$ in Eqs. (18) and (16) recovers the expressions for non-relativistic particles (Bell and Inan, 1981). We can show that similarly to the non-relativistic case, the conservation of magnetic moment results in magnetic trapping. Assuming a slab geometry for the magnetic field as shown in Eq. (6) for $z \sim s_{\|}$, i.e., the component along the parallel coordinate, and the magnetic field of the form $B\left(s_{\|}\right)=B_{0}(1+$ $\frac{9}{2} s_{\|}^{2} / R^{2}$ ) to mimic the dipolar field, for which $R$ is equal to the Earth's radius, we can solve both Eqs. (18) and (16). Hence, replacing the expression for $B\left(s_{\|}\right)$in Eq. (18) results in the following equation:

$\frac{d^{2} s_{\|}}{\mathrm{d} t^{2}}+\frac{9 \mu B_{0}}{m \gamma R^{2}} s_{\|}=0$.

The solution of the above equation is therefore of the form $s_{\|} \sim \cos \left(\sqrt{\frac{9 \mu B_{0}}{m \gamma R^{2}}} t\right)$. Replacing the expression for the magnetic field in Eq. (16) for the perpendicular momentum, we find the following differential:

$$
\frac{\mathrm{d} p_{\perp}}{p_{\perp}}=\frac{9}{2 R^{2}} d\left(s_{\|}^{2}\right)
$$

with a perpendicular momentum solution

$$
p_{\perp}=p_{\perp 0} e^{\left(\frac{9}{2 R^{2}} s_{\|}^{2}\right)} .
$$

Replacing the solution for $s_{\|}$in the above equation provides for a complete solution for the particle motion in the inhomogeneous field $B\left(s_{\|}\right)=B_{0}\left(1+\frac{9}{2} s_{\|}^{2} / R^{2}\right)$. We see that the particle oscillates back and forth along the parallel direction, while the perpendicular momentum increases as the particle reaches regions of larger magnetic field strength corresponding to $s_{\|} \sim R$.

\subsection{Surfatron breaking due to inhomogeneous B field effect}

Numerous effects can cause the breaking of the surfatron process: dispersive wave effects, dissipation of the wave amplitude, inhomogeneous magnetic fields damping the acceleration along the field line, or simply the result of precipitation into the atmosphere. Because of the slow time scales upon which the surfatron process becomes interesting to sustain particle precipitation, and since other effects would take place on longer time scales, we now focus solely on the inhomogeneous effect. That is, we want to obtain time scales for which the surfatron would not be prevented by field inhomogeneities.

The surfatron results in the parallel acceleration of a particle caused by the parallel component of the electric field. As demonstrated in the previous section, magnetic field inhomogeneities result in a $-\mu \nabla B$ force that can reduce the surfatron process, in the same way that the parallel electric field from a longitudinal component can prevent parallel acceleration. Hence, when $e \delta E_{\|} \sim \mu \nabla B$, parallel acceleration becomes marginal. This condition translates as follows:

$\frac{s_{\|}}{R} \sim \frac{\delta_{1}}{n} \frac{\Omega_{0} R}{\left(\gamma_{0}^{2}-1\right) \sin \left(\alpha_{0}\right)^{2} c}$ 


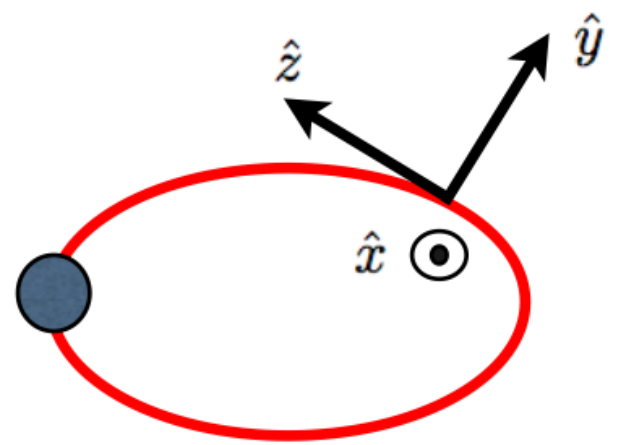

Fig. 6. Slab geometry for the dipolar magnetic field.

for the parameters $\delta_{1}=\delta B / B_{0}$ and $n=c / v_{\phi}$, the gyrofrequency $\Omega_{0}=e B_{0} / m c$ as well as the pitch angle $\alpha_{0}$ and Lorentz factor $\gamma_{0}$ of the particle at the equatorial region. Setting $n=3, \delta_{1} \sim 0.01, R \sim 6000 \mathrm{~km}, \Omega_{0} \sim 3 \times 10^{4}$ for a particle with an initial energy of the order of $100 \mathrm{keV}$ and pitch angle of $45^{\circ}$, we find $s_{\|} \sim 10^{4} \mathrm{~km}$. Therefore, we can conclude that a particle would gain energy of the order of $W \sim e \delta E_{\|} s_{\|}$, which corresponds to a gain in energy of the order of $100 \mathrm{keV}$ for an electron interacting with an electric field of $100 \mathrm{mV} \mathrm{m}^{-1}$. This approximation is comparable to energization of electrons reported by Artemyev et al. (2013) who found gains of the order of $80-100 \mathrm{keV}$ for particles going through several Landau resonance in an inhomogeneous field. The difference with our result hereafter, however, is that the particle gains energy during the time of one Landau resonance, making the process much more efficient, even though less probable.

We now write a dynamical system for a relativistic charged particle interacting with an obliquely propagating wave in an inhomogeneous field. In order to make the set of equations more transparent to the reader, we write them in a coordinate axis for which $\hat{z} \| \hat{b}$, that is the background magnetic field is parallel to the $z$ axis. We denote $\hat{y}=\hat{\perp}_{1}$ and $\hat{x}=\hat{\perp}_{2}$. Rewriting the magnetic field in terms of this coordinate system, we obtain:

$$
\left\{\begin{array}{l}
\delta B_{x}=\delta B \sin (\Phi) \\
\delta B_{y}=\delta B \cos (\Phi) \cos (\theta) \\
\delta B_{\|}=-\delta B \cos (\Phi) \sin (\theta)
\end{array}\right.
$$

and similarly, using Faraday's laws, we obtain the following components for the electric field:

$$
\left\{\begin{array}{l}
\delta E_{x}=-v_{\Phi} \delta B \cos (\Phi) / c \\
\delta E_{y}=v_{\Phi} \delta B \sin (\Phi) \cos (\theta) / c \\
\delta E_{\|}=-v_{\Phi} \delta B \sin (\Phi) \sin (\theta) / c
\end{array}\right.
$$

for the phase $\Phi=k_{\|} z+k_{\perp 1} y-\omega t=k z-k y-\omega t$. We choose the background magnetic field to be written as $\mathbf{B}_{0}=-y B_{0} g^{\prime}(z) \hat{y}+B_{0} g(z) \hat{z}$, for the function $g(z)=1+z^{2} / R^{2}$ and its partial derivative with respect to $z, g^{\prime}(z)$, denoting the background magnetic field variation
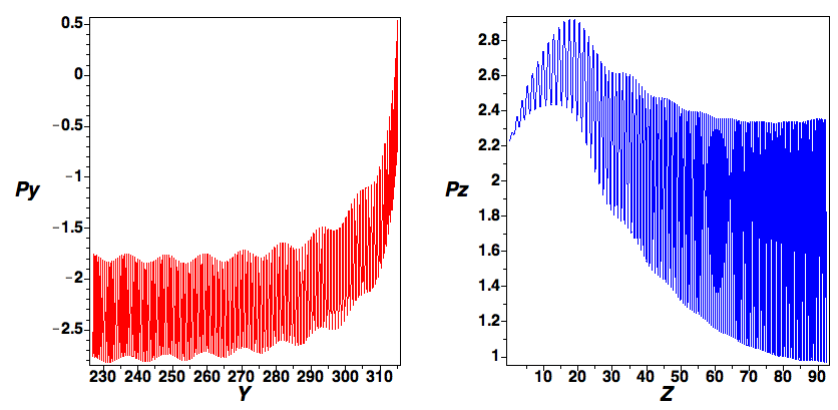

Fig. 7. Orbits for parameters $\delta_{1}=0.01, v_{\phi}=0.33 c, \delta_{2}=0.1$ and $\theta=71.9^{\circ}$. The left panel shows the perpendicular component of momentum $P_{y}$ against the perpendicular position $Y$. The right panel shows the parallel component of momentum $P_{Z}$ against the parallel position $Z$. Initially the parallel component of momentum increases because of surfatron, until the $-\mu \nabla B$ force becomes sufficiently strong to break the trapping due to the parallel electric field. The particle gains energy of the order of $76 \mathrm{keV}$ during the process.

as the particle propagates along the field line and away from the equatorial region $z \sim 0$. We can then write the dynamical system in terms of the variables $\left(p_{x}, p_{y}, p_{z}, z\right.$, $\left.y, \Omega_{0}=e B_{0} / m \gamma c, \Omega_{1}=e \delta B / m \gamma c\right)$ and the functions $g(z)$ and $q(y, z)=y g^{\prime}(z)$ :

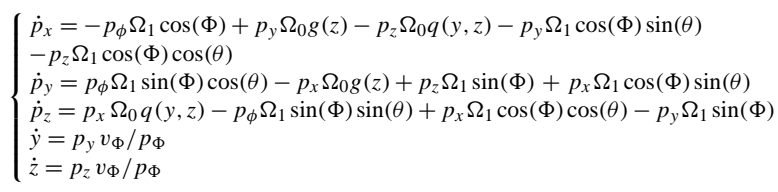

and

$$
\begin{aligned}
\dot{\Omega}_{0} & =\frac{\mathrm{d}}{\mathrm{d} t}\left(\frac{e B_{0}}{m c \gamma}\right) \\
& =-\left(\frac{e B_{0}}{m c \gamma}\right) \frac{1}{\gamma} \frac{\mathrm{d} \gamma}{\mathrm{d} t} \\
& =-\Omega_{0} \frac{p \dot{p}}{m^{2} \gamma^{2} c^{2}} \\
& =-\Omega_{0} \frac{p c^{2}}{m^{2} c^{4}+p^{2} c^{2}} \dot{p} \\
& =\frac{-\Omega_{0} \Omega_{1} p_{\Phi}}{m^{2} \gamma^{2} c^{2}}\left(\sin (\Phi)\left(p_{y} \cos (\theta)-p_{z} \sin (\theta)\right)-p_{x} \cos (\Phi)\right) .
\end{aligned}
$$

We then proceed by normalizing the variables as follows: $p_{i} / m v_{\phi}=P_{i}, k z=Z, k y=Y, \Omega_{0} / \omega=\delta_{3}$ and $\omega t=\tau$, and write the dynamical system in terms of the normalized variables $P_{x}, P_{y}, P_{z}, Y, Z$, and $\delta_{3}$ and the parameters $\delta_{1}, \delta_{2}$ and $n$ as previously defined.

$$
\left\{\begin{array}{l}
\dot{P}_{x}=-\delta_{1} \cos (\Phi) / \delta_{2}+P_{y} \delta_{3} g(Z)-P_{z} \delta_{3} q(Y, Z)-P_{y} \delta_{1} \delta_{3} \cos (\Phi) \sin (\theta) \\
-P_{z} \delta_{1} \delta_{3} \cos (\Phi) \cos (\theta) \\
\dot{P}_{y}=\delta_{1} \sin (\Phi) \cos (\theta) / \delta_{2}-P_{x} \delta_{3} g(Z)+P_{z} \delta_{1} \delta_{3} \sin (\Phi)+P_{x} \delta_{1} \delta_{3} \cos (\Phi) \sin (\theta) \\
\dot{P}_{z}=P_{x} \delta_{3} q(Y, Z)-\delta_{1} \sin (\Phi) \sin (\theta) / \delta_{2}+P_{x} \delta_{1} \delta_{3} \cos (\Phi) \cos (\theta)-P_{y} \delta_{1} \delta_{3} \sin (\Phi)(27) \\
\dot{Y}=\delta_{2} \delta_{3} P_{y} \\
\dot{Z}=\delta_{2} \delta_{3} P_{z} \\
\dot{\delta_{3}}=-\frac{\delta_{1} \delta_{2} \delta_{3}^{3}}{n^{2}}\left(\sin (\Phi)\left(P_{y} \cos (\theta)-P_{z} \sin (\theta)\right)-P_{x} \cos (\Phi)\right)
\end{array}\right.
$$

We can now integrate this dynamical system for parameters relevant to radiation belt electrons with large-amplitude 
obliquely propagating waves to study the surfatron process in an inhomogeneous magnetic field mimicking the Earth's magnetic field. Figure 8 show orbits for parameters $\delta_{1}=0.01$, $v_{\phi}=0.33 c, \delta_{2}=0.1$ and $\theta=71.9^{\circ}$. The left panel shows the perpendicular component of momentum $P_{y}$ against the perpendicular position $Y$. The right panel shows the parallel component of momentum $P_{z}$ against the parallel position $Z$. Initially the parallel component of momentum increases because of surfatron, until the $-\mu \nabla B$ force becomes sufficiently strong to break the trapping due to the parallel electric field. The particle gains energy of the order of $76 \mathrm{keV}$ during the process. This gain in energy is of similar order $(\sim 100 \mathrm{keV})$ as the one computed above for a simple balance of forces. Even though this gain appears modest, it should be kept in mind that it takes place on a time scale of the order of $\tau \sim 12$, hence $t \sim 10 \mathrm{~ms}$ for a wave frequency $\omega \sim 3 \mathrm{kHz}$. As the surfatron acceleration is lost, the conservation of the adiabatic invariant leads to a transfer of energy from the parallel direction to the perpendicular as noted by the continuous increase of the perpendicular momentum for $\tau>12$. Since the gain in energy is irreversible, the particle uplifted by tens of $\mathrm{keV}$ can only oscillate back and forth in the potential of the wave and transfer energy along the perpendicular or parallel direction to preserve adiabatic invariance.

This point is clearly demonstrated in the right panel of Fig. 8. As the particle is uniformly accelerated, $\gamma$ increases. Once the surfatron is broken, the particle resides in a state of higher energy. The left panel of the figure shows the threedimensional orbit in velocity space $V x, V y, V z$. For $\tau<12$, the particle is uniformly accelerated through the surfatron process along the parallel direction. Once the surfatron is made inoperable, the magnetic field gradient dictates the particle orbits and conservation of $\mu$ leads to transfer of energy to the perpendicular direction. As denoted by the left panel of Fig. 8, the gyroradius of the particle increases $\left(V_{y} \gg V_{z}\right)$ as the particle comes out of the surfatron.

Even though the surfatron accelerates particles parallel to the magnetic field, the inhomogeneous field results in redistributing the energy perpendicularly to the magnetic field. Hence, such a process, if statistically common in the radiation belts, could provide for an explanation to anisotropic distribution resulting in whistler wave turbulence without the need to resort to cyclotron resonance. Indeed, both resonant and nonresonant electron whistler instabilities require an initial anisotropy with $K_{\perp}$, the perpendicular kinetic energy density, to exceed $K_{\|}$, the parallel kinetic energy density by a certain amount. As the waves are being triggered by the instability and $K_{\|} / K_{\perp}$ reaching marginal stability levels, particles in the tail can be accelerated through the surfatron, travel toward a region of larger magnetic field and gain greater gyroradius, bounce back to equatorial region and contribute to the breaking of the marginal stability state of the whistler turbulence. This back and forth mechanism could then be stopped by precipitating the particles in the atmosphere, instead of having them bounce back toward the equatorial region.
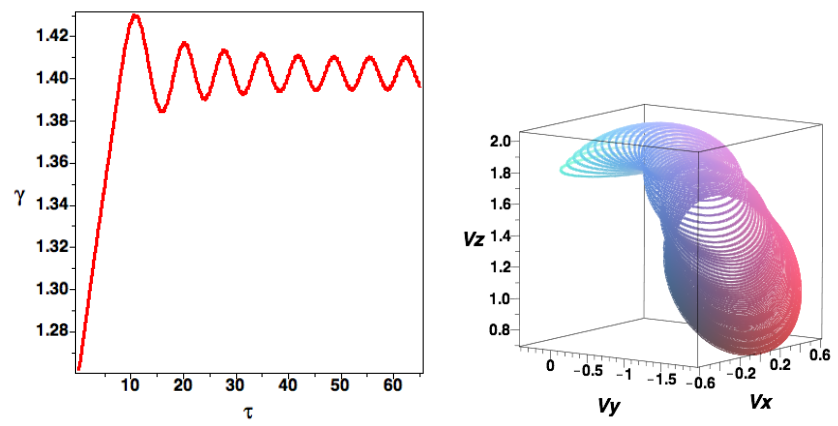

Fig. 8. Orbits for parameters $\delta_{1}=0.01, v_{\phi}=0.33 c, \delta_{2}=0.1$ and $\theta=71.9^{\circ}$. The left panel shows the relativistic Lorentz factor $\gamma$ against normalized time $\tau=\omega t$. The right panel shows the threedimensional orbit in velocity space $V x, V y, V z$. For $\tau<12$, the particle is uniformly accelerated through the surfatron process along the parallel direction. Once the surfatron is made inoperable, the magnetic field gradient dictates the particle orbits and conservation of $\mu$ leads to transfer of energy to the perpendicular direction. The gyroradius of the particle increases $\left(V_{y} \gg V_{z}\right)$ as the particle comes out of the surfatron.

\section{Conclusions}

We extended a previous theoretical study (Osmane and Hamza, 2012b) of nonlinear wave-particle interactions for the study of electrons in radiation belts by including longitudinal wave effects and inhomogeneous magnetic fields. We found that, similarly than for the electromagnetic case in a uniform background field, the acceleration of particles along the background magnetic field, for propagation angles in close proximity to a critical propagation $\theta_{c}$ and associated with a Hopf-Hopf bifurcation condition, can arise on sufficiently small timescales to be of relevance to radiation belt dynamics. Even though longitudinal wave components and inhomogeneous magnetic fields can limit the surfatron acceleration of electrons in radiation belts, gains in energy of the order of $100 \mathrm{keV}$, taking place on one tenth of a millisecond, are sufficiently strong for the mechanism to sustain particle energization on timescales of the order of $0.1 \mathrm{~ms}$. Future studies will investigate the effect of wave obliquity and field inhomogeneities on electron distribution functions for parameters consistent with radiation belt dynamics.

\section{Appendix A}

\section{Dynamical system derivation for a transverse electromagnetic wave}

Our starting point is the relativistic Lorentz equation for the motion of a particle in an electromagnetic field. The force is therefore written as

$$
\frac{\mathrm{d} \boldsymbol{p}}{\mathrm{d} t}=e\left[\boldsymbol{E}(\boldsymbol{x}, t)+\frac{\boldsymbol{v}}{c} \times \boldsymbol{B}(\boldsymbol{x}, t)\right]
$$


for a particle of momentum $\boldsymbol{p}=m \gamma \boldsymbol{v}$, rest mass $m$ and charge $e$. The Lorentz contraction factor $\gamma$ is defined as follows:

$\gamma=\frac{1}{\sqrt{1-\frac{v^{2}}{c^{2}}}}$.

In order to avoid dealing with both the velocity and the momentum in the dynamical system, we simply write the equations in terms of the more physically relevant quantity of the two, that is the relativistic momentum $p$ :

$$
\frac{\mathrm{d} \boldsymbol{p}}{\mathrm{d} t}=e\left[\boldsymbol{E}(\boldsymbol{x}, t)+\frac{\boldsymbol{p}}{m \gamma c} \times \boldsymbol{B}(\boldsymbol{x}, t)\right] .
$$

Similarly, the Lorentz factor can be written as follows:

$$
\gamma=\frac{\sqrt{m^{2} c^{4}+p^{2} c^{2}}}{m c^{2}}
$$

The electromagnetic field is superposed onto a background magnetic field $B_{0}$.

$$
\begin{aligned}
& \boldsymbol{E}(\boldsymbol{x}, t)=\delta \boldsymbol{E}(\boldsymbol{x}, t) \\
& \boldsymbol{B}(\boldsymbol{x}, t)=\boldsymbol{B}_{0}+\delta \boldsymbol{B}(\boldsymbol{x}, t)
\end{aligned}
$$

The electromagnetic wave vector points in the $\hat{z}$ direction and the background magnetic field lies in the $y-z$ plane.

$$
\begin{aligned}
& \boldsymbol{k} \cdot \boldsymbol{B}_{0}=\boldsymbol{k} B_{0} \cos (\theta) \\
& \left\{\begin{array}{l}
\delta \boldsymbol{E}=\delta E_{x} \hat{\boldsymbol{x}}+\delta E_{y} \hat{\boldsymbol{y}} \\
\delta \boldsymbol{B}=\delta B_{x} \hat{\boldsymbol{x}}+\delta B_{y} \hat{\boldsymbol{y}}
\end{array}\right.
\end{aligned}
$$

where

$$
\left\{\begin{array}{l}
\delta B_{x}=\delta B \sin (k z-\omega t) \\
\delta B_{y}=\delta B \cos (k z-\omega t)
\end{array} .\right.
$$

Faraday's law, expressed in terms of the Fourier components gives the relation between the components of the electromagnetic fields.

$c \boldsymbol{k} \times \delta \boldsymbol{E}(\boldsymbol{k}, \omega)=\omega \delta \boldsymbol{B}(\boldsymbol{k}, \omega)$

The electric force is therefore written as

$$
\left\{\begin{array}{l}
F_{E x}=e v_{\phi} \delta B \cos (k z-\omega t) / c \\
F_{E y}=-e v_{\phi} \delta B \sin (k z-\omega t) / c \\
F_{E z}=0
\end{array}\right.
$$

for which $v_{\phi}=\omega / k$ is the phase velocity. Taking the cross product of the momentum and the magnetic field, the magnetic force is written as

$$
\left\{\begin{array}{l}
F_{B x}=\frac{1}{m \gamma^{c}}\left(p_{y} e B_{0} \cos (\theta)+p_{z} e B_{0} \sin (\theta)-p_{z} e \delta B_{y}\right) \\
F_{B y}=\frac{1}{m \gamma^{c}}\left(-p_{x} e B_{0} \cos (\theta)+p_{z} e \delta B_{x}\right) \\
F_{B z}=\frac{1}{m \gamma c}\left(-p_{x} e B_{0} \sin (\theta)+p_{x} e \delta B_{y}-p_{y} e \delta B_{x}\right)
\end{array} .\right.
$$

We can write the dynamical system equations in terms of the following variables: $p_{\Phi}=m \gamma v_{\Phi} ; \Omega_{1}=e \delta B / m c \gamma$; $\Omega_{0}=e B_{0} / m c \gamma$, which results in the following equations:

$\left\{\begin{array}{l}\dot{p}_{x}=p_{y} \Omega_{0} \cos (\theta)+\left(p_{\Phi}-p_{z}\right) \Omega_{1} \cos (k z-\omega t)+p_{z} \Omega_{0} \sin (\theta) \\ \dot{p}_{y}=-p_{x} \Omega_{0} \cos (\theta)+\left(p_{z}-p_{\Phi}\right) \Omega_{1} \sin (k z-\omega t) \\ \dot{p}_{z}=-p_{x} \Omega_{0} \sin (\theta)+p_{x} \Omega_{1} \cos (k z-\omega t)-p_{y} \Omega_{1} \sin (k z-\omega t) \\ \dot{z}=p_{z} v_{\Phi} / p_{\Phi}\end{array}\right.$

In the classical case we have 4 equations to integrate, the three components of the velocity plus the position coordinate along $\boldsymbol{k}$. In the relativistic case, for a non-zero propagation angle, the energy of the particle is not a constant of the motion, that is, $\dot{\gamma} \neq 0$. Hence, we can think of the relativistic dynamical system as composed of 4 equations, the three components of the momentum plus the position coordinate along $\boldsymbol{k}$, and one constraint relating $\gamma$ and the momentum components. Without any loss of generality we take the constraint into consideration by writing an equation for the dynamical gyrofrequency:

$$
\begin{aligned}
\dot{\Omega}_{0} & =\frac{\mathrm{d}}{\mathrm{d} t}\left(\frac{e B_{0}}{m c \gamma}\right) \\
& =-\left(\frac{e B_{0}}{m c \gamma}\right) \frac{1}{\gamma} \frac{\mathrm{d} \gamma}{\mathrm{d} t} \\
& =-\Omega_{0} \frac{p \dot{p}}{m^{2} \gamma^{2} c^{2}} \\
& =-\Omega_{0} \frac{p c^{2}}{m^{2} c^{4}+p^{2} c^{2}} \dot{p} \\
& =-\frac{\Omega_{0} \Omega_{1} p_{\Phi}}{m^{2} \gamma^{2} c^{2}}\left(p_{x} \cos (k z-\omega t)-p_{y} \sin (k z-\omega t)\right) .
\end{aligned}
$$

If we define the constant $\delta=\Omega_{1} / \Omega_{0}$, it is straightforward to see that

$\dot{\Omega}_{1}=\delta \dot{\Omega}_{0}$.

Since $p_{\Phi}=p_{\Phi}(\gamma)$, the time evolution of this quantity is written as:

$$
\begin{aligned}
\dot{p}_{\Phi} & =m v_{\Phi} \frac{\mathrm{d} \gamma}{\mathrm{d} t} \\
& =m v_{\Phi} \frac{p}{\gamma m^{2} c^{2}} \dot{p} \\
& =-m v_{\Phi} \gamma \frac{\dot{\Omega}_{0}}{\Omega_{0}} .
\end{aligned}
$$

We can now eliminate the explicit time dependence of the equations by making a transformation of variables. Even though this transformation corresponds to a translation in the wave frame for low phase speed of the wave $\left(v_{\phi} \ll c\right)$, it does not correspond to a physical frame of reference for phase speeds similar to the speed of light $v_{\phi} \sim c^{2}$. The explicit time dependence can therefore be eliminated by the following change of variables:

\footnotetext{
${ }^{2}$ The transformation in the position coordinates along $z$ and $v_{z}$ are Lorentz transformations in the wave frame, but because we do not also transform the time component into the wave frame $\left(t^{\prime}=\gamma\left(t+v_{\phi} z / c^{2}\right)\right.$, the dynamical system does not correspond to a particle orbit in the wave frame for relativistic regimes.
} 
$p_{x}^{\prime}=p_{x}, p_{y}^{\prime}=p_{y}, p_{z}^{\prime}=\gamma_{w}\left(p_{z}-p_{\phi}\right), z^{\prime}=\gamma_{w}\left(z-v_{\phi} t\right)$

for the Lorentz factor:

$$
\gamma_{w}=\frac{1}{\sqrt{1-\frac{v_{\Phi}^{2}}{c^{2}}}} .
$$

We can then write the equations of motion in terms of the new variables as follows:

$$
\left\{\begin{array}{l}
\dot{p}_{x}^{\prime}=\Omega_{0} p_{y}^{\prime} \cos (\theta)-\Omega_{1} p_{z}^{\prime} \cos \left(k z^{\prime} / \gamma_{w}\right) / \gamma_{w}+\Omega_{0}\left(p_{z}^{\prime} / \gamma_{w}+p_{\phi}\right) \sin (\theta) \\
\dot{p}_{y}^{\prime}=-\Omega 0 p_{x}^{\prime} \cos (\theta)+\Omega_{1} p_{z}^{\prime} \sin \left(k z^{\prime} / \gamma_{w}\right) / \gamma_{w} \\
\dot{p}_{z}^{\prime} / \gamma_{w}=-\Omega_{0} p_{x}^{\prime} \sin (\theta)+\Omega_{1} p_{x}^{\prime} \cos \left(k z^{\prime} / \gamma_{w}\right)-\Omega_{1} p_{y}^{\prime} \sin \left(k z^{\prime} / \gamma_{w}\right)-\dot{p}_{\Phi}
\end{array}\right.
$$

If we absorb the Lorentz factor $\gamma_{w}$ into $p_{z}^{\prime}$ and $k$, that is, we write $p_{z}^{\prime} \rightarrow p_{z}^{\prime} / \gamma_{w}$ and $k \rightarrow k / \gamma_{w}$, and write $\dot{p}_{\Phi}$ in terms of $\left(p_{x}^{\prime}, p_{y}^{\prime}, p_{z}^{\prime}, z^{\prime}, \Omega_{0}\right)$, we can write the dynamical system as follows:

$$
\left\{\begin{array}{l}
\dot{p}_{x}^{\prime}=\Omega_{0} p_{y}^{\prime} \cos (\theta)-\Omega_{1} p_{z}^{\prime} \cos \left(k z^{\prime}\right)+\Omega_{0}\left(p_{z}^{\prime}+p_{\phi}\right) \sin (\theta) \\
\dot{p}_{y}^{\prime}=-\Omega_{0} p_{x}^{\prime} \cos (\theta)+\Omega_{1} p_{z}^{\prime} \sin \left(k z^{\prime}\right) \\
\dot{p}_{z}^{\prime}=-\Omega_{0} p_{x}^{\prime} \sin (\theta)+\Omega_{1}\left(\frac{n^{2}-1}{n^{2}}\right)\left(p_{x}^{\prime} \cos \left(k z^{\prime}\right)-p_{y}^{\prime} \sin \left(k z^{\prime}\right)\right) \\
\dot{z}^{\prime}=p_{z}^{\prime} v_{\Phi} / p_{\Phi}
\end{array}\right.
$$

with the refractive index $n^{2}=c^{2} / v_{\Phi}^{2}$. The magnitude of the momentum is now written as $p^{\prime}=\sqrt{p_{x}^{\prime 2}+p_{y}^{\prime 2}+\left(p_{z}^{\prime} / \gamma_{w}\right)^{2}}$, hence the Lorentz contraction factor also transforms from $\gamma(p) \rightarrow \gamma\left(p^{\prime}\right)$.

Acknowledgements. This work was supported by the Natural Sciences and Engineering Research Council of Canada (NSERC). One of the authors, A. M. Hamza, wishes to acknowledge CSA (Canadian Space Agency) support. Computational facilities are provided by ACEnet, the regional high-performance computing consortium for universities in Atlantic Canada.

Edited by: T. Hada

Reviewed by: P. Yoon and G. S. Lakhina

\section{References}

Albert, J. M.: Nonlinear interaction of outer zone electrons with VLF waves, Geophys. Res. Lett., 29, 116-1-116-3, doi:10.1029/2001GL013941, 2002.

Artemyev, A. V., Krasnoselskikh, V. V., Agapitov, O. V., Mourenas, D. and Rolland, G.: Non-diffusive resonant acceleration of electrons in the radiation belts, Phys. Plasmas, 19, 122901, doi:10.1063/1.4769726, 2013.

Bell, T. F. and Inan, U.: Transient nonlinear pitch angle scattering of energetic electrons by coherent vlf wave packets in the magnetosphere, J. Geophys. Res., 86, 9047-9063, 1981.

Cattell, C., Wygant, J. R., Goetz, K., Kersten, K., Kellogg, P. J., von Rosenvinge, T., Bale, S. D., Roth, I., Temerin, M., Hudson, M. K., Mewaldt, R. A., Wiedenbeck, M., Maksimovic, M., Ergun, R., Acuna, M., and Russell, C. T.: Discovery of very large amplitude whistler-mode waves in Earth's radiation belts, Geophys. Res. Lett. 35, L01105, doi:10.1029/2007GL032009, 2008.
Chen, Y., Reeves, G. D., and Friedel, R. H. W.: The energization of relativistic electrons in the outer van allen radiation belt, Nature, 3, 614-617, 2007.

Friedel, R. H. W., Reeves, G. D., and Obara, T.: Relativistic electron dynamics in the inner magnetosphere - a review, J. Atmos. Sol.Terr. Phy., 64, 265-282, 2002.

Green, J. C. and Kivelson, M. G.: Relativistic electrons in the outer radiation belt: Differentiating between acceleration mechanisms, J. Geophys. Res., 109, A03213, doi:10.1029/2003JA010153, 2004.

Horne, R. B., Thorne, R. M., Shprits, Y. R., Merredith, N. P., Glauert, S. A., Smith, A. J., Kanekal, S. G., Baker, D. N., Engebretson, M. J., Posch, J. L., Spasojevic, M., Inan, U. S., Pickett, J. S., and Decreau, P. M.: Wave acceleration of electrons in the van allen radiation belts, Nature, 437, 227-230, 2005.

Kellogg, P. J., Cattell, C. A., Goetz, K., Monson, S. J., and Wilson III, L. B.: Electron trapping and charge transport by large amplitude whistlers, Geophys. Res. Lett., 37, L20106, doi:10.1029/2010GL044845, 2010.

Kersten, K., Cattell, C. A., Breneman, A., Goetz, K., Kellogg, P. J., Wygant, J. R., Wilson III, L. B., Blake, J. B., Looper, M. D., and Roth, I.: Observation of relativistic electron microbursts in conjunction with intense radiation belt whistler-mode waves, Geophys. Res. Lett., 38, 8107, doi:10.1029/2011GL046810, 2011.

Kulsrud, R. M.: Plasma physics for astrophysics, Princeton University Press, New Jersey, 2005.

Lorentzen, K. R., Blake, J. B., Inan, U. S., and Bortnik, J.: Observations of relativistic electron microburts in association with vlf chorus, J. Geophys. Res., 106, 6017-6027, 2001.

Miyoshi, Y., Morioka, A., and Obara, T.: Large enhancementof the outer belt electrons during magnetic storms, Earth Planets Space, 53, 1163-1170, 2002.

O’brien, T. P., Lorentzen, K. R., Mann, I. R., Meredith, N. P., Blake, J. B., Fennell, J. F., Looper, M. D., Milling, M. K., and Anderson, R. R.: Energization of relativistic electrons in the presence of ulf power and mev microbursts: Evidence for dual vlf and vlf acceleration, J.Geophys. Res., 108, 1329, doi:10:1029/202JA009784, 2003.

Omura Hikishima, M. and Summers, D.: Microburst precipitation of energetic electrons associated with chorus wave generation, Geophys. Res. Lett., 37, L07103, doi:10.1029/2010GL042678, 2010.

Osmane, A. and Hamza, A. M.: Relativistic acceleration of Landau resonant particles as a consequence of Hopf bifurcations, Phys. Plasmas, 19, 030702, doi:10.1063/1.3692234, 2012a.

Osmane, A. and Hamza, A. M.: Dynamical-systems approach to relativistic nonlinear wave-particle interaction in collisionless plasmas, Phys. Rev. E, 85, 056410, doi:10.1103/PhysRevE.85.056410, 2012b.

Shprits, Y. Y., Thorne, R. M., Horne, R. B., Glauert, S. A., Cartwright, M., Russell, C. T., Baker, D. N., and Kanekal, S. G.: Acceleration mechanism responsible for the formation of the new radiation belt during the 2003 halloween solar storm, J. Geophys. Res., 33, L05104, doi:10.1029/2005GL024256, 2006.

Summers, D. and Omura, Y.: Ultra-relativistic acceleration of electrons in planetary magnetospheres, Geophys. Res. Lett., 34, 24205, doi:10.1029/2007GL032226, 2007. 
Thorne, R. M., Horne, B., Glauert, S., Meredith, N., Shprits, Y. Y., Ss, D., and Anderson, R.: The influence of wave-particle interactions on relativistic electron dynamics during storms, in: vol. Inner Magnetosphere Interactions: New Perspectives From Imaging, Geophysical Monograph 159, edited by: Burch, J., Schulz, M., and Spence, H., American Geophysical Union, Washington, D.C., 2005.
Wilson III, L. B., Cattell, C. A., Kellogg, P. J., Wygant, J. R., Goetz, K., Breneman, A., and Kersten, K.: The properties of large amplitude whistler mode waves in the magnetosphere: Propagation and relationship with geomagnetic activity, Geophys. Res. Lett., 38, L17107, doi:10.1029/2011GL048671, 2011.

Yoon, P. H.: Large-amplitude whistler waves and electron acceleration, Geophys. Res. Lett., 38, L12105, doi:10.1029/2011GL047893, 2011. 